\title{
Assessment of the insecticidal activity of afoxolaner against Aedes aegypti in dogs treated with NexGard ${ }^{\circledR}$
}

\author{
Julian Liebenberg ${ }^{1}$, Josephus Fourie $^{1}$, Wilfried Lebon ${ }^{2}$, Diane Larsen $^{2}$, Lenaïg Halos ${ }^{2}$, and Frédéric Beugnet ${ }^{2, *}$ \\ ${ }^{1}$ Clinvet International (Pty) Ltd, PO Box 11186, 9321 Universitas, South Africa \\ ${ }^{2}$ Boehringer Ingelheim Animal Health, 29 avenue Tony Garnier, 69007 Lyon, France
}

Received 21 August 2017, Accepted 5 October 2017, Published online 23 October 2017

\begin{abstract}
Twelve healthy dogs were studied in this parallel group, blinded, randomised, and negative controlled efficacy study. On Day -1, the 12 dogs included were ranked within sex in descending order of individual pretreatment (Day -5) fed mosquito counts and randomly allocated by blocks of two dogs to the untreated control group or the afoxolaner-treated group. NexGard ${ }^{\circledR}$ (Merial, now part of Boehringer Ingelheim Animal Health) was administered orally on Day 0 in accordance with the European label instructions. On Days 1, 7, 14, 21 and 28, all dogs were exposed for a duration of 1 hour to $50 \pm 5$ unfed Aedes aegyptifemales. After each exposure, mosquitoes were collected after 1 hour and assessed for viability during collection and at $24 \pm 2$ hours. The arithmetic (and geometric) mean values of live fed mosquito counts at 24 hours after the exposure periods for the negative control group ranged from 33.7 (32.3) to 49.8 (49.7), indicating that this was a vigorous mosquito strain. There was no significant difference between control and treated groups in the number of live and fed mosquitoes at each 1 hour post-exposure collection time. Based on arithmetic and geometric mean values at 24 hours after each exposure, significantly fewer live fed mosquitoes were recorded in the treated group, compared to the negative control group, throughout the study $(p<0.001)$. The afoxolaner insecticidal efficacy against A. aegypti varied from $98 \%$ (Day 2$)$ to $75.3 \%$ (Day 29) based on arithmetic means, and 98.7\% (Day 2) to 89.8\% (Day 29) based on geometric means.
\end{abstract}

Keywords: Aedes aegypti, insecticide, afoxolaner, NexGard ${ }^{\circledR}, \operatorname{dog}$

Résumé- Évaluation de l'activité insecticide de l'afoxolaner contre Aedes aegypti chez les chiens traités avec NexGard ${ }^{\circledR}$. Douze chiens en bonne santé ont été étudiés dans cette étude d'efficacité en aveugle, en groupes parallèles et avec contrôles négatifs. Au jour -1, les 12 chiens inclus ont été classés par sexe par ordre décroissant de prétraitement individuel (jour -5) de comptage de moustiques nourris et répartis au hasard par blocs de deux chiens, en groupe témoin non traité et en groupe traité par afoxolaner. Du NexGard ${ }^{\circledR}$ (Merial, maintenant un membre de Boehringer Ingelheim Animal Health) a été administré par voie orale au jour 0 conformément aux instructions dela notice européenne. Aux jours 1, 7, 14, 21 et 28, tous les chiens ont été exposés pendant une durée de 1 heure à $50 \pm 5$ Aedes aegypti femelles à jeun. Une heure après chaque exposition, les moustiques ont été recueillis et leur viabilité a été évaluée à la collecte et après $24 \pm 2$ heures. Les valeurs moyennes arithmétiques (et géométriques) du nombre de moustiques vivants et nourris 24 heures après les périodes d'exposition pour le groupe témoin négatif variaient de 33,7 (32,3) à 49,8 (49,7), ce qui indique une bonne viabilité de la souche de moustiques. Il n'y avait pas de différence significative entre les groupes témoins et les groupes traités dans le nombre de moustiques vivants et nourris à chacune des collectes à 1 heure post-exposition. Sur la base des valeurs moyennes géométriques et arithmétiques, à 24 heures après chaque exposition, des nombres significativement plus petits de moustiques nourris et vivants ont été enregistrés dans le groupe traité par rapport au groupe témoin non traité pendant toute l'étude $(p<0,001)$. L'efficacité insecticide de l'afoxolaner contre A. aegypti variait de $98 \%$ (jour 2) à 75,3\% (jour 29) sur la base de moyennes arithmétiques, $98,7 \%$ (jour 2) à 89,8\% (jour 29) sur la base de moyennes géométriques.

\section{Introduction}

Recently, a new class of insecticides/acaricides, the isoxazolines, have demonstrated very good efficacy

\footnotetext{
*Corresponding author: Frederic. beugnet@merial.com
}

against fleas and ticks [19]. Afoxolaner is an isoxazoline administered monthly to protect dogs against fleas and ticks (NexGard ${ }^{\circledR}$, Merial, now part of Boehringer Ingelheim Animal Health) $[2,3,8,10]$. It is administered at a minimum dose of $2.5 \mathrm{mg} / \mathrm{kg}$. Recent studies have demonstrated its activity against other arthropods, including 
Demodex canis, the agent of canine demodicosis, Sarcoptes scabiei var. canis and S. scabiei var. suis, the agent of sarcoptic mange in dogs and swine, respectively, as well as Otodectes cynotis, the agent of ear mange in dogs and cats $[1,4,5]$.

After oral administration, afoxolaner is absorbed quickly, with peak plasma levels (Cmax) reached between 2 to 4 hours after administration [14,15]. Plasma protein binding is more than $99 \%$, which explains the long half-life, $10-14$ days on average $[14,15]$. Due to its strong binding to plasma proteins, its activity is systemic and exposure is related to the ingestion of blood or inflammatory fluids by the biting insect.

In addition to its activity against well-known bloodfeeding ectoparasites like fleas and ticks, or resident ectoparasites like Demodex, Sarcoptes, and Otodectes, it is probable that afoxolaner would also have a certain level of insecticidal activity against other blood-feeding arthropods like mosquitoes. Insecticidal efficacy following a blood meal might not prevent pathogen transmission from the female mosquito, but it could have a further effect by killing the mosquitoes before a new bite, and/or by reducing the mosquito population in a restricted area like a household where treated dogs are living. It could therefore have an indirect action on the rate of vector-borne pathogen transmission within the household.

Aedes aegypti mosquitoes are endemic in tropical areas around the globe, but have expanded into sub-tropical areas and even some warm temperate locations, although the species seems less adaptable to temperate climate than Aedes albopictus [13]. It is now found in many parts of the world including South and Central America, the southern USA, Africa, India, tropical islands, South-East Asia, Northern Australia, and sporadically in the Mediterranean zone [13]. A. aegypti is a major vector of several diseases of animals and/or humans, e.g. heartworm disease due to Dirofilaria immitis in dogs, equine encephalitis viruses, West Nile virus, Dengue virus, Chikungunya virus, Zika virus, and yellow fever virus $[9,12]$. The objective of this study was to assess the insecticidal activity that afoxolaner may have against $A$. aegypti mosquitoes.

\section{Materials and methods}

The design and conditions of this study were approved by the South African and ClinVet animal welfare ethics committees, and were performed in accordance with the Good Clinical Practices of the European Agency for the Evaluation of Veterinary Medicinal Products (CVMP/ VICH GL9, July 2000; CVMP/VICH GL19, July 2001). This study was a parallel group, blinded, randomised, negative controlled efficacy study. It was conducted with two groups of six dogs each.

Male and female dogs were included in the study if they had been acclimatised to the study conditions for at least 8 days; they were clinically healthy as verified by a veterinarian on Day -8; they were $\geq 6$ months at the time of inclusion (Day -1); females were not pregnant; they had not been treated with a long-acting topical or systemic acaricide/insecticide during the 12 weeks preceding Day 0.

The animals were kept individually in cages and no physical contact between dogs was possible. However, animals still had visual and auditory contact with conspecifics. During the acclimatisation period (Day -8 to Day -1), an initial $A$. aegypti mosquito challenge was performed on Day -5 to evaluate the receptivity of each dog to experimental infestation and for random allocation of the dogs to the study groups. The 12 dogs included in the study were randomly allocated to two groups (untreated control group and afoxolaner-treated group), based on total counts of fed mosquitoes 1 hour after the initial challenge. In addition, veterinary clinical examination was performed on Day - 8 for enrolment purposes, and weighing of all dogs was performed on Day -1 for appropriate dose determination. All the dogs were observed daily from Day -8 to Day 28 for their general health.

On Day 0, all dogs assigned to the treated group received afoxolaner. The dogs were treated orally with NexGard $^{\circledR}(2.27 \% \mathrm{w} / \mathrm{w}$ afoxolaner chewable tablets $)$ in accordance with European label instructions [8]. All dogs weighed from 10 to $25 \mathrm{~kg}$ and were treated with a chewable tablet containing $68 \mathrm{mg}$ of afoxolaner. The dogs were observed hourly for 4 hours after administration to detect possible adverse reactions.

Dogs were challenged with $50 \pm 5$ Aedes aegypti unfed female mosquitoes on Day -5 for randomisation purposes, and then on Days 1, 7, 14, 21 and 28 to assess insecticidal activity. Mosquitoes were assessed for viability and feeding status during collection 1 hour after exposure and on Days 2, 8, 15, 22 and 29 (24 hours after exposure).

To perform the mosquito challenge, the dogs were sedated using medetomidine (Domitor ${ }^{\circledR}$, Zoetis), and placed into a mosquito proof net (dimensions: $81 \mathrm{~cm} \times 58$ $\mathrm{cm} \times 58 \mathrm{~cm}$ ). The whole body of the dog was thus exposed to the mosquito challenge. The mosquito net used allowed both exposure of dogs to the parasites and collection of parasites after the challenge, without mosquitoes escaping during the process. A Clinvet laboratory-bred strain of $A$. aegypti of US origin was used in the infestation challenges.

Food was removed at least two hours prior to sedation of animals or animals were fasted overnight if required by scheduling constraints. The $50 \pm 5$ female mosquitoes were released into the net and they were carefully vacuumed after 1 hour.

At the end of the exposure period, atipamezole Antisedan $^{\circledR}$, Zoetis) was used to reverse the effects of the sedation in dogs.

One hour after challenge, the mosquitoes were collected using an aspirator and they were then assessed as live, fed or unfed, moribund or dead. Mosquitoes were classified as live if they exhibited normal behaviour and were capable of coordinated locomotion and flight upon external stimuli. Mosquitoes were classified as moribund if they were only capable of some movement, but exhibit abnormal, obviously impaired behaviour, and were not capable of coordinated locomotion or flight upon external stimuli. 
Table 1. Comparison of body weights between dogs and mosquito counts obtained $1 \mathrm{~h}$ after exposure at Day -5 for allocation purposes.

\begin{tabular}{|c|c|c|c|c|}
\hline & Day & Statistic & Control dogs & Afoxolaner-treated dogs \\
\hline Body weight (kg) & Day -1 & $\mathrm{n}$ & 6 & 6 \\
\hline \multirow[t]{5}{*}{$p$-value: 0.8655} & & Mean & 16.37 & 16.03 \\
\hline & & $\mathrm{SD}$ & 2.467 & 3.999 \\
\hline & & Median & 16.00 & 14.70 \\
\hline & & Minimum & 13.8 & 12.4 \\
\hline & & Maximum & 19.2 & 23.6 \\
\hline Mosquito count $1 \mathrm{~h}$ after exposure & Day -5 & $\mathrm{n}$ & 6 & 6 \\
\hline \multirow[t]{6}{*}{$p$-value: 0.5008} & & Mean & 53.3 & 54.0 \\
\hline & & $\mathrm{SD}$ & 2.25 & 0.63 \\
\hline & & GeoMean & 53.3 & 54.0 \\
\hline & & Median & 53.0 & 54.0 \\
\hline & & Minimum & 50 & 53 \\
\hline & & Maximum & 57 & 55 \\
\hline
\end{tabular}

p-value: One-way ANOVA with a treatment effect.

Prior feeding by dead mosquitoes was assessed following the collection, by placing the dead mosquito on tissue paper and squashing the abdomen with a spatula or similar suitable object to assess if a blood meal was taken.

Live and moribund mosquitoes were incubated in suitable containers at $24.3^{\circ} \mathrm{C}$ to $28.1^{\circ} \mathrm{C}$ for 24 hours ( \pm 2 hours). During this period the mosquitoes had access to a $10 \%$ sucrose solution, or a suitable alternative. The mosquitoes were again assessed for viability following the 24-hour ( \pm 2 hours) incubation period and then assessed for feeding as described above. All live and moribund mosquitoes were immobilised in a freezer prior to the feeding assessments.

The dead mosquito counts observed after each challenge are the sum of the dead mosquitoes counted at 1 hour (Table 1) and the dead mosquitoes counted at 24 hours (Table 2).

Insecticidal activity calculations were based on both arithmetic and geometric mean values. Geometric mean efficacy calculations were based on the geometric mean values of the mosquito (count +1 ) data. One (1) was subsequently subtracted from the result to obtain a meaningful value for the geometric mean of each group.

The primary efficacy of afoxolaner against $A$. aegypti mosquitoes was calculated using the total live fed mosquitoes at 24 hours after each mosquito challenge, according to the formula below:

Insecticidal efficacy (\%) against mosquitoes $=100 \mathrm{x}$ $(\mathrm{Mc}-\mathrm{Mt}) / \mathrm{Mc}$, where:

$\mathrm{Mc}=$ mean number of live fed mosquitoes in the control group at 24 hours after challenge;

$\mathrm{Mt}=$ mean number of live fed mosquitoes in the treated group 24 hours after challenge.

The groups were compared using an ANOVA (Proc GLM procedure in SAS) with a treatment effect on both untransformed and logarithmic transformed mosquito $($ count +1$)$ data. SAS Version 9.3 TS Level 1M2 was used for all the statistical analyses.

\section{Results}

The weight of dogs varied from 13.8 to $19.2 \mathrm{~kg}$ in the control group $($ mean $=16.37 \mathrm{~kg})$ and from 12.4 to $23.6 \mathrm{~kg}$ (mean value $16.02 \mathrm{~kg}$ ) in the treated group. No statistically significant differences were recorded between the pretreatment fed mosquito counts at Day $-5(p=0.5008)$ nor the body weights $(p=0.8655)$ of the dogs in the two groups, which indicated homogeneity between the dogs included in each group (Table 1).

No adverse events were recorded after treatment or during the study duration [6].

The live, moribund and dead status of the mosquitoes was assessed at all time-points (Tables 2 and 3). The collection of live mosquitoes at 1 hour post-exposure indicated a mortality of 1.86 to $8 \%$ during the contact time between dogs and mosquitoes (Table 2). There was no significant difference between the control group and the treated group in the numbers of live or dead mosquitoes at the end of the 1 hour exposure (Table 2). No moribund mosquitoes were observed at $1 \mathrm{~h}$.

The arithmetic mean values of live fed mosquito counts at 24 hours after the challenge period for the negative control group ranged from 33.7 to 49.8 , indicating that this was a vigorous mosquito strain. The insecticidal and acaricidal activities, following the European Medicine Agency guideline (EMA) [7] and the World Association for the Advancement of Veterinary Parasitology (WAAVP) [16], should be based on the comparison of the number of live arthropods collected from control and treated animals (Table 3). Arithmetic and geometric mean values of live fed mosquito counts and efficacies are summarised in Tables 3 and 4.

Based on both arithmetic and geometric mean values of live fed mosquitoes at 24 hours, significantly fewer live fed mosquitoes were recorded for the afoxolaner treated group compared to the negative control group, throughout the study $(p<0.001)$. Based on arithmetic and geometric mean values of live fed mosquitoes at 24 hours, the insecticidal efficacy of NexGard ${ }^{\circledR}$ was $98.2 \%$ (98.7\% based 


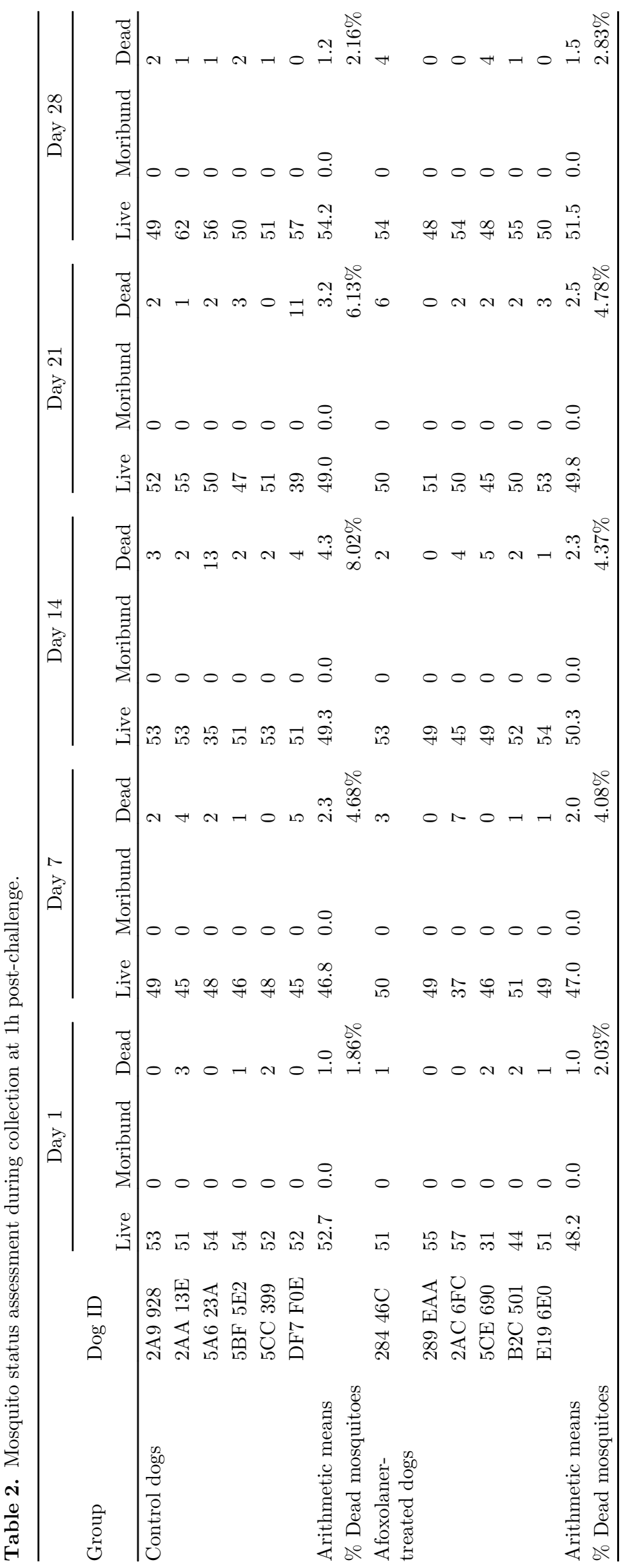




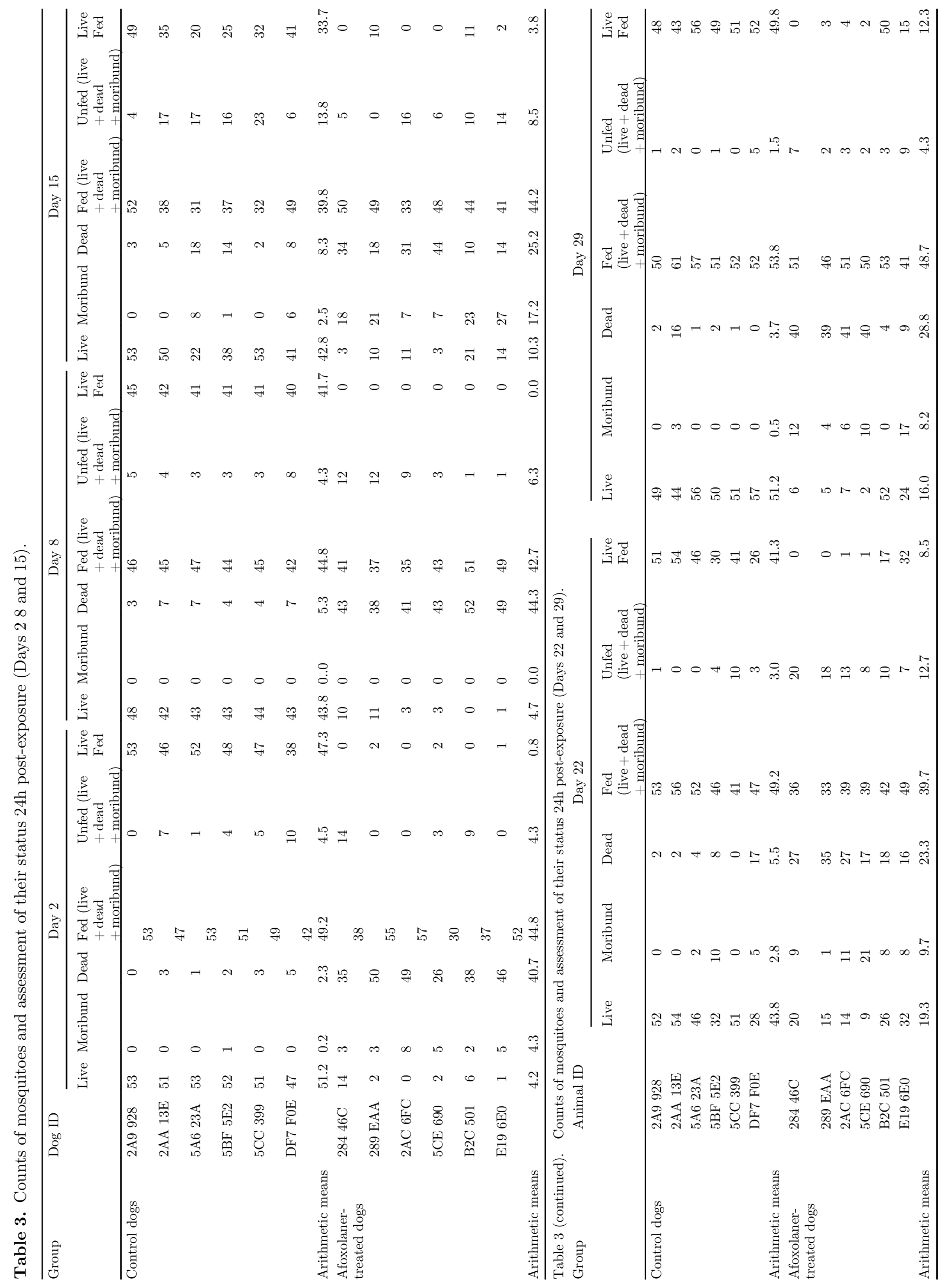


Table 4. Insecticidal efficacy based on live fed mosquitoes counted at 24 hours post-exposure.

\begin{tabular}{lllllr}
\hline & \multicolumn{2}{c}{ Control Group } & & \multicolumn{2}{c}{ Afoxolaner-treated Group } \\
\cline { 2 - 2 } Day & $\begin{array}{l}\text { Arithmetic (Geometric) } \\
\text { Mean }\end{array}$ & & $\begin{array}{l}\text { Arithmetic (Geometric) } \\
\text { Mean }\end{array}$ & $\begin{array}{l}\text { Percentage efficacy } \\
\text { (based on geometric means) }\end{array}$ & $\begin{array}{l}\text { ANOVA } \\
p \text {-Value }\end{array}$ \\
\hline Day 2 & $47.3(47.1)$ & $0.8(0.6)$ & $98.2(98.7)$ & $<0.0001$ \\
Day 8 & $41.7(41.6)$ & $0.0(0.0)$ & $100.0(100.0)$ & $<0.0001$ \\
Day 15 & $33.7(32.3)$ & $3.8(1.7)$ & $88.6(94.7)$ & 0.0001 \\
Day 22 & $41.3(40.0)$ & $8.5(2.7)$ & $79.4(93.4)$ & 0.0005 \\
Day 29 & $49.8(49.7)$ & $12.3(5.0)$ & $75.3(89.8)$ & 0.001 \\
\hline
\end{tabular}

on geo mean), $100 \%$ (100\%), $88.6 \%$ (94.7\%), $79.4 \%$ $(93.4 \%)$, and $75.3 \%(89.8 \%)$, on days $2,8,15,22$ and 29 , respectively (Table 4$)$.

\section{Discussion and conclusion}

The study design classically used to assess repellency of insecticides after $1 \mathrm{~h}$ of exposure of flying insects was used in this particular study to assess insecticidal activity after feeding [16]. Being systemic, afoxolaner binds to plasma proteins [15], and no repellent activity related to volatile molecules on the skin surface was expected. The number of fed mosquitoes observed at $1 \mathrm{~h}$ was not different between the control and treated dogs ( $>90 \%$ in both groups), thus confirming the absence of a repellent effect, which is assessed by the anti-feeding effect. No differences in the number of live and dead mosquitoes were observed at $1 \mathrm{~h}$ collection, indicating that there was no immediate killing effect.

The insecticidal activity was high 24 hours after each exposure challenge, indicating that the A. aegypti mosquitoes ingested a lethal dose of afoxolaner during their blood meal. The blood meal of female $A$. aegyptitakes only a few minutes $(<5 \mathrm{~min})$ and the volume ingested is about $4-5 \mu \mathrm{L}[9,11,17,18]$. The study design based on 1 hour exposure to dogs allowed more than $90 \%$ of mosquitoes to feed and it is expected that the maximum proportion was reached in such a period. After a single administration at Day 0 , the quantity of afoxolaner present in $4-5 \mu \mathrm{L}$ of dog blood was enough to kill $>75 \%$ (>89\% in geometric mean) of the fed female mosquitoes throughout an entire month. There are currently no data on specific mosquito species sensitivity and these results should be confirmed in other important species such as Culex pipiens or A. albopictus.

Given the lack of anti-feeding effect, it is not expected that afoxolaner treatment in dogs would have a direct impact on the transmission of pathogenic agents by $A$. aegypti during the blood meal. Nevertheless, A. aegypti females need a blood meal every 2-3 days, and 48 hours is needed for oviposition $[9,11,18]$. This species is also unlikely to disperse and has restricted flight capacity, estimated to be less than $500 \mathrm{~m}[9,11]$. The behaviour tends to be indoor. These biological aspects are in favour of a rapid decrease of the mosquito population biting treated dogs. A female would die after its blood meal and would not bite a second time; neither would the insect be able to lay eggs before dying. This hypothesis would need to be demonstrated under a simulated household environment, but the results of this study are in favour of an indirect protective effect in households where afoxolaner-treated dogs are living. Killing Aedes females before a new blood meal would reduce the rate of transmission of vector-borne pathogens, like heartworm in dogs.

Acknowledgements. The authors would like to thank Ms $\mathrm{H}$ Jooste for the statistical analysis.

\section{Conflict of interest}

This clinical study was funded by Merial, now part of Boehringer Ingelheim Animal Health, 29 Avenue Tony Garnier, 69007 Lyon of which Frédéric Beugnet, Lénaïg Halos and Wilfried Lebon are employees.

ClinVet, of which Julian Liebenberg and Josephus Fourie are employees, is an independent South African Contract Research Organisation contracted to conduct the study.

All authors voluntarily publish this article and have no personal interest in these studies other than publishing the scientific findings that they have been involved in generating via planning, initiating, monitoring and conducting the investigations and analysing the results.

\section{Disclaimer}

NEXGARD ${ }^{\circledR}$ is a registered trademark of Merial SAS. All other brands are the property of their respective owners.

This document is provided for scientific purposes only. Any reference to a brand or a trademark herein is for informational purposes only and is not intended for a commercial purpose or to dilute the rights of the respective owner(s) of the brand(s) or trademark(s).

\section{References}

1. Beugnet F, de Vos C, Liebenberg J, Halos L, Larsen D, Fourie J. Efficacy of afoxolaner in a clinical field study in dogs naturally infested with Sarcoptes scabiei. 2016. Parasite, 23, 26. 
2. Beugnet F, Liebenberg J, Halos L. 2015. Comparative efficacy of two oral treatments for dogs containing either afoxolaner or fluralaner against Rhipicephalus sanguineus sensu lato and Dermacentor reticulatus. Veterinary Parasitology, 209, 142-145.

3. Beugnet F, Liebenberg J, Halos L. 2015. Comparative speed of efficacy against Ctenocephalides felis of two oral treatments for dogs containing either afoxolaner or fluralaner. Veterinary Parasitology, 207, 297-301.

4. Beugnet F., Halos L., Larsen D., de Vos C. Efficacy of oral afoxolaner for the treatment of canine generalised demodicosis. Parasite 2016, 23, 14.

5. Carithers D, Crawford J, de Vos C, Lotriet A, Fourie J. Assessment of afoxolaner efficacy against Otodectes cynotis infestations of dogs. 2016. Parasites \& Vectors, 9, 635.

6. Drag M, Saik J, Harriman J, Larsen D. 2014. Safety evaluation of orally administered afoxolaner in 8-week-old dogs. Veterinary Parasitology, 201, 198-203.

7. European Medicine Agency Committee for Medicinal Products for Veterinary Use. 2007. Guidelines for the Testing and Evaluation of the Efficacy of Antiparasitic Substances for the Treatment and Prevention of Tick and Flea Infestation in Dogs and Cats. EMA Guideline No. EMEA/CVMP/EWP/005/2000-Rev2-200\%. London, 2007.

8. European Medicines Agency. Nexgard ${ }^{\circledR}$ summary of product characteristics. 2014 http:/ / www.ema.europa.eu/docs/ en GB/document library/EPAR - Product Informa tion/veterinary/002729/WC5001640 $\overline{7} \overline{7}$.pdf

9. Foster W.A., Walker E.D. 2009. Chapter 14: Mosquitoes, in Medical and Veterinary Entomology Second Edition, Edited by G.R. Mullen and L.A. Durden, Elsevier, London, 207-259.

10. Halos L, Lebon W, Chalvet-Monfray K, Larsen D, Beugnet F. 2014. Immediate efficacy and persistent speed of kill of a novel oral formulation of afoxolaner (NexGard ${ }^{\circledR}$ ) against induced infestations with Ixodes ricinus ticks. Parasites and Vectors, 7, 452.

11. Kettle DS. 1995. Culicidae (Mosquitoes), in Medical and Veterinary Entomology, Ed CAB International, Oxon, UK, 109-151.
12. Kock R.A. 2015. Vertebrate reservoirs and secondary epidemiological cycles of vector borne diseases. Revue Scientifique et Technique (International Office of Epizootics), 34, 151-153.

13. Kraemer M, Sinka ME, Duda KA, Mylne AQN, Shearer FM, Barker CM, Moore CG, Carvalho RG, Coelho GO, Van Bortel W, Hendrickx G, Schaffner F, Elyazar IRF, Teng HJ, Brady HJ, Messina JP, Pigott DM, Scott TW, Smith DL, Wint WGR, Golding N, Hay SI. 2015. The global distribution of the arbovirus vectors Aedes aegypti and Ae. albopictus. eLife, 4, e08347.

14. Letendre L, Harriman J, Drag M, Mullins A, Malinski T, Rhebein S. 2016. The intravenous and oral pharmacokinetics of afoxolaner and milbemycin oxime when used as a combination chewable parasiticide for dogs. Journal of Veterinary Pharmacology and Therapeutics, 40, 35-43

15. Letendre L, Harriman J, Huang R, Kvaternick V, Drag M, Larsen DL. 2014. The intravenous and oral pharmacokinetics of afoxolaner, a novel isoxazoline, used as a monthly chewable antiparasitic for dogs. Veterinary Parasitology, 201, 190-197.

16. Marchiondo AA, Holdsworth PA, Fourie LJ, Rugg D, Hellmann K, Snyder DE, Dryden MW. 2013. World Association for the Advancement of Veterinary Parasitology (W.A.A.V.P.) second edition: guidelines for evaluating the efficacy of parasiticides for the treatment, prevention and control of flea and tick infestations on dogs and cats. Veterinary Parasitology, 194, 84-97.

17. Rodhain F. 2015. Insects as vectors: systematics and biology. Revue Scientifique et Technique (International Office of Epizootics), 34, 83-96.

18. Russell RC, Otranto D, Wall R. 2013. Mosquitoes (Diptera: Culicidae), in The Encyclopedia of Medical and Veterinary Entomology, Ed CAB International, Wallingford, UK, 243-282.

19. Shoop WL, Hartline EJ, Gould BR, Waddell ME, McDowell RG, Kinney JB, Lahm, GP, Long JK, Xu M, Wagerle T, Jones GS, Dietrich RF, Cordova D, Schroeder ME, Rhoades DF, Benner EA, Confalone PN. 2014. Discovery and mode of action of afoxolaner, a new isoxazoline parasiticide for dogs. Veterinary Parasitology, 201, 179-189.

Cite this article as: Liebenberg J, Fourie J, Lebon W, Larsen D, Halos L, Beugnet F. 2017. Assessment of the insecticidal activity of afoxolaner against Aedes aegypti in dogs treated with NexGard ${ }^{\circledR}$. Parasite 24, 39

\section{PARASTE}

Reviews, articles and short notes may be submitted. Fields include, but are not limited to: general, medical and veterinary parasitology; morphology, including ultrastructure; parasite systematics, including entomology, acarology, helminthology and protistology, and molecular analyses; molecular biology and biochemistry; immunology of parasitic diseases; host-parasite relationships; ecology and life history of parasites; epidemiology; therapeutics; new diagnostic tools.

All papers in Parasite are published in English. Manuscripts should have a broad interest and must not have been published or submitted elsewhere. No limit is imposed on the length of manuscripts.

Parasite (open-access) continues Parasite (print and online editions, 1994-2012) and Annales de Parasitologie Humaine et Comparée (1923-1993) and is the official journal of the Société Française de Parasitologie. 